\title{
Original Surgical Repair for an Adult Pectus Excavatum Patient: A Case Report
}

\author{
Ryuta Fukai ${ }^{1}$, Hiroshi Iida ${ }^{2}$ and Yoshihito Irie $^{3}$
}

Department of General Thoracic Surgery, Shonan Kamakura General Hospital, 1370-1, Okamoto, Kamakura, Japan

Department of Cardiovascular and Chest Wall Surgery, Nagoya Tokusyukai General Hospital, 2-52, Kozojicyo-kita, Kasugai, Japan

Department of Cardiovascular Surgery, Iwaki Kyoritsu General Hospital, 16, Uchigo-miyamamachi-kusehara, Iwaki, Japan

\section{Abstract}

Backgroud: Pectus excavatum, the most common congenital thoracic deformity, is particularly difficult to correct in adults with reduced chest wall flexibility. For the repair of pectus excavatum, we have conducted sterno-costal elevation, which consists of resecting the redundant costal cartilages and the lower part of the sternum and resuturing the remaining costal cartilages to the sternum, and does not use any exogenous materials or require a second operation.

Methods: We treated a 27-year-old female patient with an asymmetrical chest concavity who was referred to us because of consistent precordial pain with sterno-costal elevation. In this case, we added an oblique osteotomy across the anterior sternal table to reform the torsion of the sternum with a pneumatic powered drill.

Results: The procedure corrected the deformity well, and the patient was very satisfied with the result one year after the operation.

Conclusions: Sterno-costal elevation for pectus excavatum, which does not require any exogenous materials or additional operations, is effective in the treatment of pectus excavatum in a female adult patient with a symmetrical chest deformity and moderate sternal torsion.

\section{Introduction}

Pectus excavatum (PE) is the most common thoracic deformity. The deformity is a congenital anomaly in which the body of the sternum is depressed to form a concavity, the depth of which is commonly maximal just above the xiphisternal junction. In adult PE patients with asymmetrical deformities, correction is quite difficult because the chest wall is less flexible than in younger patients. The Nuss procedure is the prevailing method for correcting PE, but it uses exogenous materials and requires a second operation to remove the pectus bar [1]. Moreover, reports indicate that the Nuss procedure has a relatively high incidence of complications [2]. We have surgically repaired PE with stero-costal elevation (SCE), which does not employ exogenous substances or require additional surgery. The procedure consists of resecting the redundant costal cartilages and the lower part of the sternum and resuturing the remaining costal cartilages to the sternum [3]. Recently, we have used our experience with real clinical cases to develop a modified SCE procedure (SCE IV) for adult $\mathrm{PE}$ patients with asymmetrical thoracic deformities and moderate torsion of the sternum. We report the case of a 27-year-old female PE patient treated with SCE IV.

\section{Case Report}

A 27-year-old woman was referred to us because of precordialgia that had been consistently present since her high school days. Computed tomography (CT) scans showed an asymmetrical chest deformity with an excavation (Figure1). The three- dimensional CT scan revealed moderate sternal torsion (Figure2). For the purpose of improving her chest deformity and symptoms, we decided to perform an operation.

The patient underwent the surgery under general anesthesia with epidural anesthesia. Under the inframammary incision, the pectoris and rectus muscles were exfoliated from the deformed sternum and costal cartilage. Next, a section of the third to the seventh costal

\section{Publication History:}

Received: August 16, 2016

Accepted: June 10, 2017

Published: June 13, 2017

\section{Keywords:}

Chest wall, Pectus excavatum, Adult patient, Surgery, Sternocostal elevation cartilages on the right side, a section of the fourth to seventh on the left side (from 20 to $50 \mathrm{~mm}$ ), and the lower part of the sternum below the sixth cartilage were resected. Moreover, we performed an oblique osteotomy across the anterior bone cortex from the right third intercostal space to the left fourth intercostal space using a pneumatic powered drill (Surgairtome $\mathrm{II}^{\bullet}$, Zimmer Inc., Indiana, USA) with a width of $2-3 \mathrm{~mm}$ to elevate the concave right lower portion of the sternum. We then added some resutures using non-absorbable threads to fix the partially incised sternum. All the cartilage stumps were reattached to the sternum using \#3 braided polyester sutures, with the resultant force causing the sternum to rise anteriorly. After the correction of the chest wall, the inner layer of the pectoral muscle and rectus muscle were sutured together to cover the field.

The postoperative course was uneventful, and the patient was discharged on the seventh postoperative day. The symmetry of her thorax remained intact one year after operation, making the patient very satisfied with the surgical result (Figure 3 ).

\section{Discussion}

$\mathrm{PE}$ is the most common congenital chest deformity. [4] The correction of the chest wall is more difficult in adult PE patients than in younger PE patients because reduced chest wall flexibility in adult patients creates conditions where correcting the deformity requires more force [5]. To treat our adult female PE patient with an

"Corresponding Author: Dr. Ryuta Fukai, Department of General Thoracic Surgery, Shonan Kamakura General Hospital, 1370-1, Okamoto, Kamakura, Kanagawa, 247-8533, Japan; E-mail: r_fukai@shonankamakura.or.jp

Citation: Fukai R, lida H, Irie Y (2017) Original Surgical Repair for an Adult Pectus Excavatum Patient: A Case Report. Int J Surg Surgical Porced 2: 119. doi: https://doi.org/10.15344/2456-4443/2017/119

Copyright: ( ) 2017 Fukai et al. This is an open-access article distributed under the terms of the Creative Commons Attribution License, which permits unrestricted use, distribution, and reproduction in any medium, provided the original author and source are credited. 


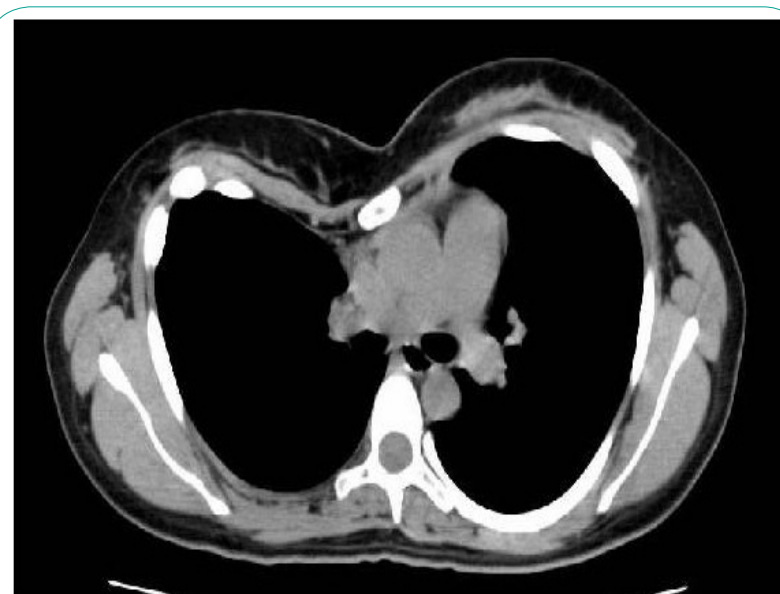

Figure 1: Regular and three-dimensional chest CT scans before surgery. They showed asymmetric pectus excavatum with tilted sternum.

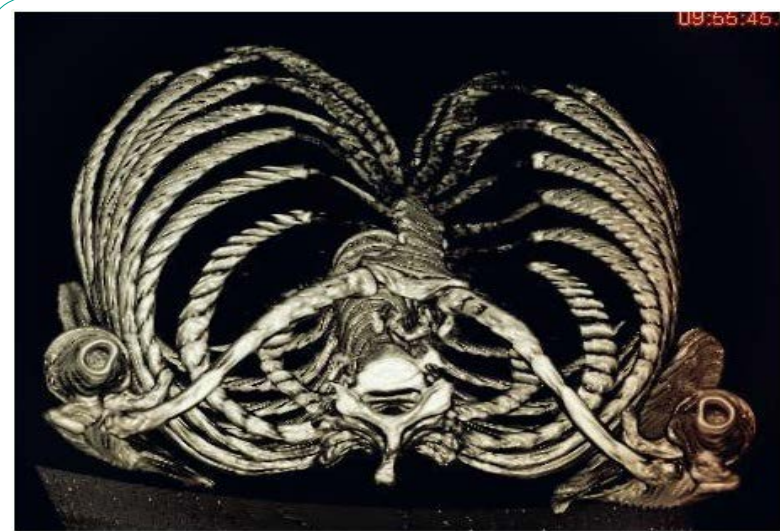

Figure 2: A three-dimensional chest osteal CT scan before surgery. It showed oblique sternal torsion.

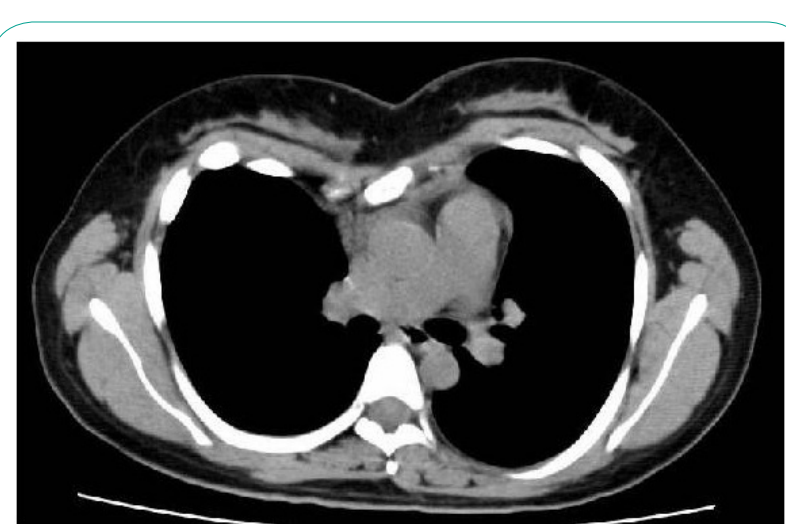

Figure 3: Regular and three-dimensional chest CT scans one year after surgery. They showed that the asymmetric deformity was corrected.

asymmetrical deformity and moderate sternal torsion, we used a modified form of the SCE procedure (SCE IV) that did not involve the use of any exogenous materials. The abnormality of the patient's thoracic contour was well corrected after the operation, making her sufficiently satisfied with the result.

Our SCE procedure was started in 1981 by Wada et al [6]. The original SCE involves resecting a section of the third to seventh redundant costal cartilages and resuturing them to the sternum under a medical vertical incision in males and an inframammary incision in females. The resected cartilages and ribs are sutured to the body of the sternum. Since these initial stages, we have worked to improve the original method. In 1993, the exfoliation of the muscles was limited to those attached to resected cartilages via a small incision (SCE II). In 1999, the lower part of the sternum below the sixth cartilage junction was resected, and the sixth and seventh cartilages were secured to the lower cutting face of the sternum (SCE III). Adequate lengths of the cartilage were resected to maintain reasonable tension to the costal cartilages: from 5 to $10 \mathrm{~mm}$ in the upper cartilages and from 30 to $50 \mathrm{~mm}$ in the lower cartilages were resected so that the resutured cartilages and ribs generated 1-3 kg (upper) and 7-12 kg (lower) of tension on the sternum. When these manipulations are applied, the sternum is pulled laterally by the shortened and resutured costal cartilages, and the sternum is raised ventrally by the resultant force. Furthermore, patients treated with SCE were allowed to start attending school or working one month after surgery and resume all types of activities, including contact sports, within three months after surgery [3].

The repair of our patient was challenging because she exhibited an asymmetrical chest deformity, moderate sternal torsion, and reduced chest wall flexibility. Therefore, we adopted SCE IV and performed an oblique anterior osteotomy from the right third intercostal space to the left fourth intercostal space to correct the angle of the sternal torsion. Furthermore, the third to seventh costal cartilages were resected on the right side, and the fourth to seventh costal cartilages were resected on the left side. This SCE IV procedure provided our patient with a smaller skin incision, shorter hospitalization, and satisfactory results. Of the 297 SCE cases we have worked on since 1993, we have performed twenty-five SCE IV procedures for adult PE cases.

The most significant merit of our procedure is that it renders second surgery unnecessary. The Nuss procedure, a surgical repair technique for PE that involves placing a metal bar under the sternum through the pleural cavities, has been associated with relatively high morbidity and recurrence rates and requires reoperation for the extraction of the metal bar [2]. Our procedure, however, is substantially uneventful and involves a short, one-time hospitalization [3]. In fact, our patient was discharged on the seventh postoperative day; slight bilateral pleural effusion was the only complication seen one month after operation, and the symmetry of the thoracic contour remained intact one year after the operation.

\section{Conclusions}

Our modified SCE procedure (SCE IV) was effective in the treatment of an adult PE patient with a symmetrical chest deformity and moderate sternal torsion. The advantages of the procedure are that it does not require any exogenous materials or additional operations.

\section{Competing Interests}

The authors declare that they have no competing interests.

\section{Acknowledgements}

We thank Kaori Matsushita, the medical clerk for industrious collecting medical information of the patient.
Int J Surg Surgical Porced

ISSN: 2456-4443
IJSSP, an open access journal Volume 2. 2017. 119 
Citation: Fukai R, Iida H, Irie Y (2017) Original Surgical Repair for an Adult Pectus Excavatum Patient: A Case Report. Int J Surg Surgical Porced 2: 119. doi: https://doi.org/10.15344/2456-4443/2017/119

Page 3 of 3

\section{Author Contributions}

All authors performed the operation of this case.

RF have written and revised this manuscript under the guidance of HI.

HI have performed and ameliorated SCE procedure for more than twenty years. $\mathrm{HI}$ is the operator of this case.

YI invited HI to this operation, and helped to draft the manuscript.

\section{References}

1. Nuss D, Kelly Jr RE, Croitoru DP, Kaze ME (1998) A 10-year review of a minimally invasive technique for the correction of pectus excavatum. $J$ Pediatr Surg 33: 545-552.

2. Watanabe A, Watanabe T, Obana T, Ohsawa H, Mawatari T, et al. (2004) The use of a lateral stabilizer increases the incidence of wound trouble following the Nuss procedure. Ann Thorac Surg 77: 296-300.

3. lida H, Sunazawa T, Ishida K, Doi A. (2010) Surgical repair of pectus excavatum not requiring exogenous implants in 113 patients. Eur $\mathrm{J}$ Cardiothorac Surg 37: 316-321.

4. Ravitch MM (1961) Operative treatment of congenital deformities of the chest. Am J Surg 101: 599-597.

5. Jaroszewski DE, Johnson K, McMahon L, Nortica D (2014) Sterna elevation before passing bars: a technique for improving visualization and facilitating minimally invasive pectus excavatum repair in adult patients. $J$ Thorac Cardiovasc Surg 147:1093-1095.

6. Wada J (1987) Chest deformity. Tokyo, Japan: Bunkyodo, 96-105 